\title{
Effect of Low FODMAP Diet and Aerobic Exercise on Irritable Bowel Syndrome Post Abdominal Surgeries
}

\author{
Mohamed Mahmoud Khalaf ${ }^{1}$, Samar Mahmoud Sharaf*1, Eid Rizk El Gammal ${ }^{2}$, Khadra Mohamed Ali ${ }^{1}$ \\ ${ }^{1}$ Department of Burn and Surgery, Faculty of Physical Therapy, Cairo University, Egypt. \\ ${ }^{2}$ Department of Oncosurgery, Faculty of Medicine, Al-Azhar University, Egypt. \\ *Corresponding author: Samar Mahmoud Sharaf, Mobile: (+20) 01004140023,
}

E-Mail: samarsharaf1993@gmail.com

\begin{abstract}
Background: Irritable bowel syndrome (IBS) is a functional gastrointestinal syndrome with an expanding worldwide incidence. Low FODMAP diet is a recently developed dietary strategy consists of limiting foods that are high fermentable, which might induce or aggravate IBS symptoms. Aerobic exercise is beneficial in reducing the risk of all types of gastrointestinal symptoms.

Objective: Investigate the effect of low FODMAP diet and aerobic exercise on irritable bowel syndrome post abdominal surgeries.

Patients and methods: Forty five female and male patients participated in this study. They were divided into three equal groups, group (A) received low FODMAP diet group (B) received aerobic exercise (walking on treadmill 25-40 minutes 3 times per week) group (C) received both low FODMAP diet and aerobic exercise. Evaluation was done preand post- 12 weeks of treatment by IBS Severity Scoring System (IBS-SSS) and IBS Quality of Life (IBS-QOL).

Results: There was significant reduction in post-treatment values of IBS-SSS and IBS-QOL in comparison to pretreatment values in the three groups, Post-treatment comparison between three groups showed significant difference in IBS-SSS and IBS-QOL, which revealed a significant reduction in IBS-SSS and IBS- QOL of group C compared to that of group A and group B.
\end{abstract}

Conclusion: combining low FODMAP diet and aerobic exercise had a greater effect on irritable bowel syndrome through reducing IBS symptoms and improving quality of life.

Keywords: Irritable bowel syndrome, Low FODMAP diet, Aerobic exercise, Post abdominal surgeries.

\section{INTRODUCTION}

Irritable bowel syndrome (IBS) is the commonest gastrointestinal condition, accounting for up to $50 \%$ of gastroenterology consultations each year ${ }^{(1)}$. It is characterized by recurrent abdominal pain and abnormal evacuation (2). IBS is most prevalent between the ages of 20 and 40, with a considerable female predominance ${ }^{(3)}$. It has been noted that people with IBS have a disproportionately high incidence of abdominal and pelvic surgeries ${ }^{(4)}$. The low FODMAPs diet limits foods that are high fermentable oligo-, di-, and monosaccharides, as well as polyols. The lowFODMAP diet is a 2-phased intervention, with strict reduction of all dietary FODMAPs followed by reintroduction of specific FODMAPs according to tolerance ${ }^{(5)}$. FODMAPs are inadequately absorbed and quickly fermented by gut bacteria, causing gas generation, bloating and pain ${ }^{(6)}$. As a result, lowering FODMAPs in the diet may be a treatment option for IBS (7). Exercise is a way to alleviate constipation, distension and for maintaining proper bowel function and reducing stresses ${ }^{(8)}$. Physical activity improves colonic transit time, gas transit, and bloating by stimulating gastrointestinal motility ${ }^{(9)}$. Finally, exercise is becoming more widely accepted as a safer and effective treatment for a variety of diseases, such as depression and irritable bowel syndrome ${ }^{(\mathbf{1 0})}$. So, this study was conducted to investigate the effect of low FODMAP diet and aerobic exercise on irritable bowel syndrome post abdominal surgeries.

\section{PATIENTS AND METHODS}

This study was conducted in Al-Tahrir general Hospital to investigate the effect of low FODMAP diet and aerobic exercise on irritable bowel syndrome postabdominal surgeries.

Forty five patients were divided equally into three groups: Group (A) included 10 females and 5 males and received low FODMAP diet, group (B) that included 11 females and 4 males and received aerobic exercise, and group (C) that included 11 females and 4 males and received low FODMAP diet and aerobic exercise.

\section{Ethical approval:}

This study which was approved by the Ethical Committee of the Faculty of Physical Therapy, Cairo University. The experimental protocol was explained in details for every patient before starting the initial assessment. Approval from all patients was formally obtained by signing a consent form. This work has been carried out in accordance with The Code of Ethics of the World Medical Association (Declaration of Helsinki) for studies involving humans.

Inclusion criteria: Age ranged between 25-40 years, body mass index not exceeding 32 , patients who 
underwent abdominal surgeries, and post-abdominal surgeries e.g. cholecystectomy, appendectomy, hernioplasty, gastric sleeve, kidney transplantation and splenectomy.

Exclusion criteria: Patients with communication disorders, overt GI bleeding, unintentional weight loss, inflammatory bowel disease or colorectal cancer, and the presence of a palpable abdominal mass or lymphadenopathy.

\section{Assessment procedures:}

- Standard weight scale was used to measure weight and height and then body mass index was calculated for each patient.

- IBS Severity Scoring System (IBS-SSS): The questionnaire consisted of five questions: abdominal pain intensity, abdominal distension, life interference, bowel habit dissatisfaction and abdominal pain frequency. It provides a total score ranging from 0 to 500, with high rates indicating more severity of symptoms ${ }^{(11)}$.

- IBS Quality of Life (IBS-QOL): The questionnaire includes 34 question concerning IBS's impact on psychological, social, and daily living activities. The scale can be subdivided into subscales such as dysphoria, activity interference, avoidance of food, health worry, image of body, social, sexual, as well as relationships ${ }^{(\mathbf{1 2})}$.

\section{Treatment procedures:}

- Low FODMAP diet: Nutritional intervention begins with avoidance of all high FODMAPs diet for a trial period of 6-8 weeks. Carbohydrates were then reintroduced for 4 weeks into the diet. Each FODMAP sub group was tested separately according to tolerance while symptoms are monitored ${ }^{(13,14)}$.

- Aerobic exercise: Using a program of exercise with intensity of maximum heart rate $50-70 \%$ and frequency of 3 times/week for 12 weeks. The session consisted of 5 minutes warm-up performed on the treadmill followed by active phase at moderate speed with no inclination for 15-30 minutes and ended by 5 minutes cool down ${ }^{(15,16)}$.

\section{Statistical analysis}

The collected data were coded, processed and analyzed using the SPSS (Statistical Package for Social Sciences) version 25 for Windows ${ }^{\circledR}$ (IBM SPSS Inc, Chicago, IL, USA). Descriptive statistics and ANOVA test were conducted for comparison of age between groups. Chi- squared test was used for comparison of sex and type of surgery distribution between groups. Normal distribution of data was checked using the Shapio-Wilk test for all variables. Levene's test for homogeneity of variances was conducted to test the homogeneity between groups. One way M ANOVA was performed to compare between groups effects on IBS-SSS and IBS-QOL. Post-hoc tests using the Tukey test were carried out for subsequent multiple comparison. Paired $t$ test was performed for comparison between pre-and post-treatment in each group. The level of significance for all statistical tests was set at $p$ $\leq 0.05$.

\section{RESULTS}

Table (1) showed that the subject characteristics of group A, B and C. There was no significant difference between groups in age, sex and type of surgery distribution $(\mathrm{p}>0.05)$.

Table (1): Basic characteristics of participants

\begin{tabular}{|c|c|c|c|c|}
\hline 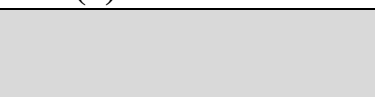 & $\begin{array}{c}\text { Group A } \\
\text { Mean } \pm \text { SD }\end{array}$ & $\begin{array}{c}\text { Group B } \\
\text { Mean } \pm \text { SD }\end{array}$ & $\begin{array}{c}\text { Group C } \\
\text { Mean } \pm \text { SD }\end{array}$ & p-value \\
\hline Age (years) & $30.73 \pm 4.41$ & $30.2 \pm 4.16$ & $31.73 \pm 4.49$ & 0.62 \\
\hline Weight (kg) & $71.13 \pm 11.52$ & $70.26 \pm 8.86$ & $73.86 \pm 11.24$ & 0.63 \\
\hline Height (cm) & $163.53 \pm 7.85$ & $166.13 \pm 8.73$ & $165.53 \pm 5.01$ & 0.6 \\
\hline BMI $\left(\mathrm{kg} / \mathrm{m}^{2}\right)$ & $26.54 \pm 3.11$ & $25.41 \pm 1.95$ & $26.98 \pm 3.52$ & 0.33 \\
\hline $\begin{array}{l}\text { Sex, n (\%) } \\
\text { Females } \\
\text { Males }\end{array}$ & $\begin{array}{c}10(67 \%) \\
5(33 \%)\end{array}$ & $\begin{array}{c}11(73 \%) \\
4(27 \%)\end{array}$ & $\begin{array}{c}11(73 \%) \\
4(27 \%)\end{array}$ & 0.89 \\
\hline Type of surgery, n (\%) & & & & \multirow{8}{*}{0.85} \\
\hline Appendectomy & $7(46.7 \%)$ & $8(53.3 \%)$ & $4(26.7 \%)$ & \\
\hline Cholecystectomy & $5(33.3 \%)$ & $5(33.3 \%)$ & $6(40 \%)$ & \\
\hline Gastric sleeve & $1(6.7 \%)$ & $0(0 \%)$ & $1(6.7 \%)$ & \\
\hline Hernioplasty & $2(13.3 \%)$ & $1(6.7 \%)$ & $2(13.3 \%)$ & \\
\hline Intestinal anastomosis & $0(0 \%)$ & $1(6.7 \%)$ & $0(0 \%)$ & \\
\hline Kidney transplantation & $0(0 \%)$ & $0(0 \%)$ & $1(6.7 \%)$ & \\
\hline Splenectomy & $0(0 \%)$ & $0(0 \%)$ & $1(6.7 \%)$ & \\
\hline
\end{tabular}

$\mathrm{SD}$, standard deviation;

$\mathrm{p}$-value, level of significance 


\section{Effect of treatment on IBS-SSS and IBS-QOL:}

Within group comparison: there was a significant reduction in IBS-SSS and IBS-QOL in the three groups posttreatment compared to pre-treatment $(\mathrm{p}<0.001)$. The percent of decrease in IBS-SSS and IBS-QOL in group A was $57.63 \%$ and $37.93 \%$ respectively and that of group B was $53.3 \%$ and $33.01 \%$ respectively, while that of group C was $69.54 \%$ and $43.75 \%$ respectively (Table $2 \&$ figures 1 and 2 ).

Between group comparison: There was no significant difference in IBS-SSS and IBS-QOL between groups' pretreatment $(p>0.05)$. Comparison between groups post-treatment showed a significant reduction in IBS-SSS of group C compared to that of group A ( $<0.01)$ and group B ( $<0.001)$. Also, there was a significant reduction in IBS- QOL of group C compared to that of group A $(\mathrm{p}<0.05)$ and group $B(p<0.01)$. There was no significant difference in IBSSSS and IBS-QOL between group A and B post-treatment ( $p>0.05)$ (Table $2 \&$ figures 1 and 2).

Table (2): Mean IBS-SSS and IBS-QOL pre- and post-treatment of group A, B and C

\begin{tabular}{|l|c|c|c|c|c|c|}
\hline & \multirow{2}{*}{$\begin{array}{c}\text { Group A } \\
\text { Mean } \pm \text { SD }\end{array}$} & Group B & Mean \pm SD & Group C & \multicolumn{3}{c|}{ Pean \pm SD } & A vs B & A vs C & B vs C \\
\cline { 5 - 7 } & & & & & & \\
\hline IBS-SSS & & & & 0.7 & 0.88 & 0.41 \\
\hline Pre-treatment & $281.66 \pm 53.84$ & $267.67 \pm 50.13$ & $290 \pm 37.55$ & 0.79 & 0.003 & 0.001 \\
\hline MD & $119.33 \pm 22.51$ & $125 \pm 27.96$ & $88.33 \pm 20.84$ & & & \\
\hline \% of change & 162.33 & 142.67 & 201.67 & & & \\
\hline t- value & 57.63 & 53.3 & 69.54 & & & \\
\hline & 12.89 & 13.84 & 19.77 & & & \\
\hline IBS-QOL & $\mathrm{p}=0.001$ & $\mathrm{p}=0.001$ & $\mathrm{p}=0.001$ & & & \\
\hline Pre-treatment & $112.66 \pm 9.95$ & $108.46 \pm 8.9$ & $110.33 \pm 8.2$ & 0.41 & 0.76 & 0.83 \\
\hline Post-treatment & $69.93 \pm 7.36$ & $72.66 \pm 9.78$ & $62.06 \pm 8.12$ & 0.65 & 0.03 & 0.004 \\
\hline MD & 42.73 & 35.8 & 48.27 & & & \\
\hline \% of change & 37.93 & 33.01 & 43.75 & & & \\
\hline t- value & 16.78 & 15.75 & 20.03 & & & \\
\hline & $\mathrm{p}=0.001$ & $\mathrm{p}=0.001$ & $\mathrm{p}=0.001$ & & & \\
\hline
\end{tabular}

$\mathrm{SD}$, Standard deviation; MD, Mean difference; $\mathrm{p}$-value, Level of significance.

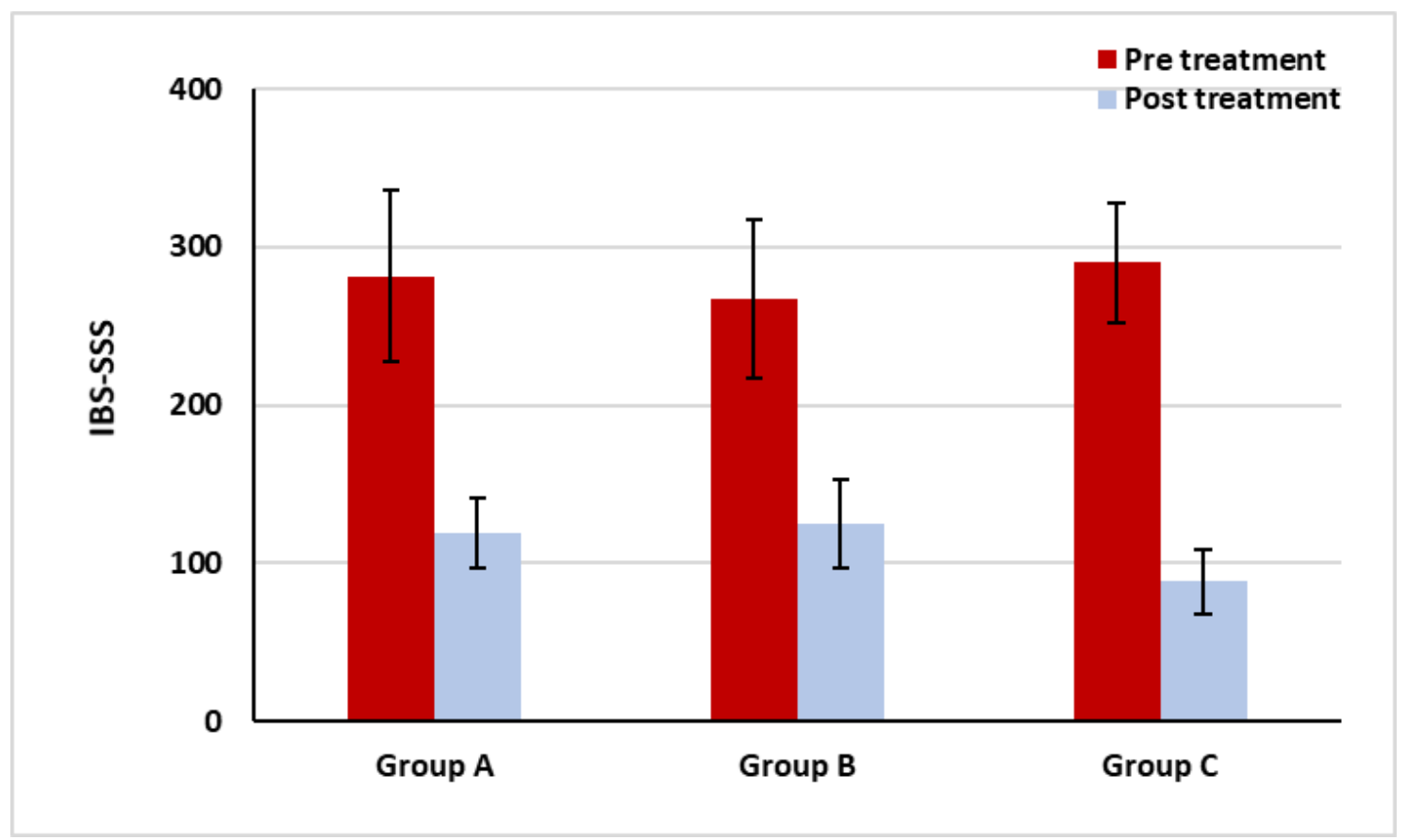

Figure (1): Mean IBS-SSS pre- and post-treatment of group A, B and C 


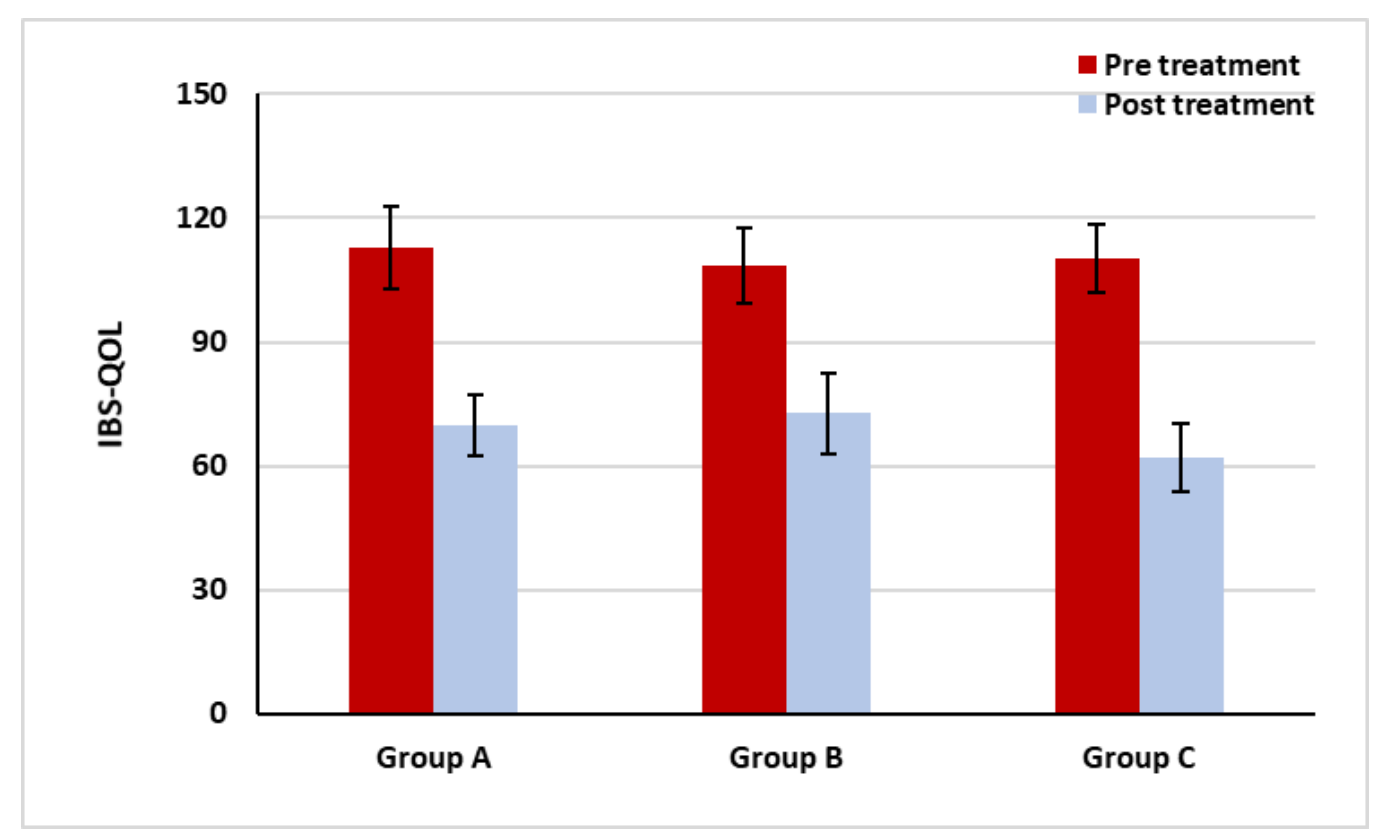

Figure (2): Mean IBS-QOL pre- and post-treatment of group A, B and C

\section{DISCUSSION}

Irritable bowel syndrome is a common disorder that has a negative influence on quality of life and causes significant health care expenditures ${ }^{(17)}$.

Our study found that Post-treatment outcomes comparison between the three groups showed a significant reduction in irritable bowel syndrome severity of symptoms and improvement in quality of life in group C compared to that of group A and group B. Outcomes of our study come in agreement with numerous clinical trials who have reported that reducing high-FODMAPs can provide adequate symptom alleviation in IBS patients ${ }^{(18,19,20)}$. According to Marsh et al. (21), those on a low FODMAP diet reported a decline in IBS -SSS scores. In addition, the IBS-QOL score showed a considerable improvement. Furthermore, it was revealed that a low FODMAP diet reduced the degree of abdominal pain, bloating, and overall symptoms.

Schumann et al. ${ }^{(22)}$ included 9 RCTs with a number of 596 subjects in their meta-analysis. In comparison with other diets. They reported that LFD was safe and effective in the short-term. O'keeffe $\boldsymbol{e t}$ al. (23) proved that low FODMAP diet is considered an effective treatment for symptoms of IBS and its effect not only for the short-term but also for long-term. Dionne et al. ${ }^{(24)}$ published a systematic review that found evidence that a low-FODMAP diet is beneficial in lowering IBS symptoms. Among the dietary therapies indicated for treating IBS symptoms, an LFD had the greatest efficacy. According to Marum et al. (25), LFD can help with gastrointestinal symptoms as well as sleep, anxiety, depression, and muscle aches.

Our findings conflict with those of Staudacher $\boldsymbol{e t}$ al. ${ }^{26)}$, who found that a low-FODMAP diet for four weeks improved IBS symptoms while lowering bifidobacteria levels, furthermore Jalanka-Tuovinen et al. (27) discovered a correlation between low bifidobacteria and IBS abdominal pain, and then a strict
low-FODMAP diet may be counterproductive. According to Halmos et al. ${ }^{(28)}$, reducing prebiotic and fermentative effects has a deleterious influence on the intestinal microenvironment, despite the fact that diet lowers IBS symptoms.

This study show improvement of IBS in physically active individuals as Zhou $\boldsymbol{e t}$ al. ${ }^{(29)}$ who mentioned that exercise is an excellent treatment for IBS since it improves bowel movement and total colon transit time. Our study agrees with Maleki et al. ${ }^{(30)}$ who noted that low-to-moderate aerobic exercise reduced the aggravation of IBS symptoms, and that this reduction was linked to reduced inflammation and increased antioxidant levels. Moreover, the results of this study come in agreement with Villoria et al. ${ }^{(31)}$ who observed the impact of aerobic exercise on abdominal bloating. Gas accumulation during rest was linked to more abdominal discomfort (3.6 score; $\mathrm{P}<$ $0.01)$, and complaints alleviated after exercise (2.8 score, $\mathrm{P}<0.05)$. This confirmed that mild regular exercise promote intestinal gas elimination and reduce bloating.

Johannesson et al. ${ }^{(32)}$ investigated the impact of aerobic activity on IBS symptoms and quality of life. They reported that long-term impacts on IBS symptoms, quality of life, fatigue, depression and anxiety were found. Fani $\boldsymbol{e t}$ al. ${ }^{(33)}$ conducted a pilot study and found that there was a substantial improvement in the intensity of IBS symptoms $(p \leq 0.001)$ and IBS quality of life $(p=0.001)$ after 6 weeks of aerobic exercise in the treadmill group compared to the control group.

On the other side our study partially conflict with results of Daley et al. ${ }^{(34)}$ who conducted RCT that investigated the efficacy and impacts of exercise on quality of life and irritable bowel symptoms. Analyses confirmed that there were no changes in quality of life scores across the groups at 12-week follow-up. The exercise group showed significant improvement of 
constipation $($ mean difference $=10.9,95 \%, \mathrm{CI}=-20.1$ -1.6) compared to usual care at follow-up.

Limitations: Firstly small sample size, which limits the generalizability of results. Secondly Psychological status of the patients that might have affected the treatment programs and evaluating procedures.

\section{CONCLUSION}

Combining a low FODMAP diet with aerobic exercise had a greater positive effect on irritable bowel syndrome post-abdominal surgeries than either a low FODMAP diet or aerobic exercise alone in reduction of symptoms and improvement quality of life.

\section{Financial support and sponsorship: Nil.}

\section{Conflict of interest: Nil.}

\section{REFERENCES}

1. Canavan C, West J, Card T (2014): The economic impact of the irritable bowel syndrome. Alimentary Pharmacology \& Therapeutics, 40 (9): 1023-34.

2. Lacy B, Mearin F, Chang L et al. (2016): Bowel disorders. Gastroenterology, 150 (6): 1393-407.

3. Gibson P, Varney J, Malakar S et al. (2015): Food components and irritable bowel syndrome. Gastroenterology, 148: 1158-1174.

4. Hasler W, Schoenfeld $P$ (2003): Abdominal and pelvic surgery in patients with irritable bowel syndrome. Alimentary Pharmacology \& Therapeutics, 17 (8): 997-1005.

5. Nanayakkara W, Skidmore P, O'Brien L et al. (2016): Efficacy of the low FODMAP diet for treating irritable bowel syndrome: the evidence to date. Clin Exp Gastroenterol., 9: 131-142.

6. Ong D, Mitchell S, Barrett J et al. (2010): Manipulation of dietary short chain carbohydrates alters the pattern of gas production and genesis of symptoms in irritable bowel syndrome. J Gastroenterol Hepatol., 25 (8): 1366-1373.

7. Bellini M, Rossi A (2018): Is a low FODMAP diet dangerous? Tech. Coloproctol., 22: 569-571.

8. Saha L (2014): Irritable bowel syndrome: Pathogenesis, diagnosis, treatment, and evidence-based medicine. World Journal of Gastroenterology, 20 (22): 6759-64.

9. Dainese R, Serra J, Azpiroz F et al. (2004): Effects of physical activity on intestinal gas transit and evacuation in healthy subjects. The American Journal of Medicine, 116 (8): 536-9.

10. Johannesson E, Simrén M, Strid H et al. (2011): Physical activity improves symptoms in irritable bowel syndrome: a randomized controlled trial. Official Journal of the American College of Gastroenterology, 106 (5): 915-22.

11. Francis C, Morris J, Whorwell P (1997): The irritable bowel severity scoring system: A simple method of monitoring irritable bowel syndrome and its progress. Aliment. Pharmacol Ther., 11: 395-402.

12. Andrae D, Patrick D, Drossman D et al. (2013): Evaluation of the Irritable Bowel Syndrome Quality of Life (IBS-QOL) questionnaire in diarrheal-predominant irritable bowel syndrome patients. Health Qual Life Outcomes, 11: 208-212.

13. Gibson $P$ (2011): Food intolerance and functional bowel disorders. J Gastroenterol Hepatol., 26 (3): 128-131.

14. Tuck C, Barrett J (2017): Re-challenging FODMAPs: the low FODMAP diet phase two. Journal of Gastroenterology and Hepatology, 32: 11-15.

15. Heyward V (2010): Advanced fitness assessment and exercise prescription. 5th ed. London: WB. Saunder Comp., Pp: 55-89.

16. Whaley M, Brubacker $P$, Oho $\mathbf{R}$ (2006): American college of sports medicine's guidelines for exercise testing and prescription. 7th ed. Philadelphia: Lippincott, Williams and Wilkins. Pp: 15690.

17. Dolan R, Chey W, Eswaran S (2018): The role of diet in the management of irritable bowel syndrome: a focus on FODMAPs. Expert Review of Gastroenterology \& Hepatology, 12 (6): 607-15.
18. Halmos E, Power V, Shepherd S et al. (2014): A diet low in FODMAPs reduces symptoms of irritable bowel syndrome. Gastroenterology, 146: 67-75.

19. Böhn L, Störsrud S, Liljebo T et al. (2015): Diet low in FODMAPs reduces symptoms of irritable bowel syndrome as well as traditional dietary advice: a randomized controlled trial. Gastroenterology, 149 (6): 1399-407.

20. Piacentino D, Rossi S, Piretta L et al. (2016): Low-FODMAPdiet in irritable bowel syndrome offers benefits not only in terms of gastrointestinal symptoms, but also in terms of psychopathology in the medium-and long-term. European Psychiatry, 33 (S1): 395399.

21. Marsh A, Eslick E, Eslick G (2016): Does a diet low in FODMAPs reduce symptoms associated with functional gastrointestinal disorders? A comprehensive systematic review and meta-analysis. Eur J Nutr., 55 (3): 897-906.

22. Schumann D, Klose P, Lauche R et al. (2015): Low fermentable, oligo-, di-, mono-saccharides and polyol diet in the treatment of irritable bowel syndrome: A systematic review and meta-analysis. Nutrition, 45: 24-31.

23. O'keeffe M, Jansen C, Martin L et al. (2018): Long-term impact of the low-FODMAP diet on gastrointestinal symptoms, dietary intake, patient acceptability, and healthcare utilization in irritable bowel syndrome. Neurogastroenterology \& Motility, 30 (1): 13154-59.

24. Dionne J, Ford A, Yuan Y et al. (2018): A Systematic Review and Meta-Analysis Evaluating the Efficacy of a Gluten-Free Diet and a Low FODMAPs Diet in Treating Symptoms of Irritable Bowel Syndrome. Am. J. Gastroenterol., 113: 1290-1300.

25. Marum A, Moreira C, Tomas-Carus $P$ et al. (2017): A low fermentable oligo-di-mono-saccharides and polyols (FODMAP) diet is a balanced therapy for fibromyalgia with nutritional and symptomatic benefits. Nutr Hosp., 34: 667-674.

26. Staudacher H, Lomer M, Anderson J et al. (2012): Fermentable carbohydrate restriction reduces luminal bifidobacteria and gastrointestinal symptoms in patients with irritable bowel syndrome. The Journal of Nutrition, 142 (8): 1510-8.

27. Jalanka-Tuovinen J, Salonen A, Nikkilä J et al. (2011): Intestinal microbiota in healthy adults: temporal analysis reveals individual and common core and relation to intestinal symptoms. PloS One, 6 (7): 230-35.

28. Halmos E, Christophersen C, Bird A et al. (2015): Diets that differ in their FODMAP content alter the colonic luminal microenvironment. Gut, 64 (1): 93-100.

29. Zhou C, Zhao E, Li Y et al. (2019): Exercise therapy of patients with irritable bowel syndrome: a systematic review of randomized controlled trials. Neurogastroenterology \& Motility, 31 (2): 13436.

30. Maleki B, Tartibian B, Mooren F et al. (2018): Low-to-moderate intensity aerobic exercise training modulates irritable bowel syndrome through antioxidative and inflammatory mechanisms in women: Results of a randomized controlled trial. Cytokine, 102: $18-25$.

31. Villoria A, Serra J, Azpiroz F et al. (2006): Physical activity and intestinal gas clearance in patients with bloating. Am J Gastroenterol., 101: 2552-2557.

32. Johannesson E, Ringström G, Abrahamsson H et al. (2015): Intervention to increase physical activity in irritable bowel syndrome shows long-term positive effects. World Journal of Gastroenterology, 21 (2): 600-605.

33. Fani M, Fani M, Chitsaz $\mathbf{N}$ et al. (2019): The effect of aerobic exercises among women with mild and moderate irritable bowel syndrome: A pilot study. Journal of Bodywork and Movement Therapies, 23: 161-165.

34. Daley A, Grimmett C, Roberts L et al. (2008): The effects of exercise upon symptoms and quality of life in patients diagnosed with irritable bowel syndrome: a randomised controlled trial. International Journal of Sports Medicine, 29 (09): 778-82. 